\title{
Marantic or Non-Bacterial Thrombotic Endocarditis
}

\author{
Viren Ahluwalia*, Walid Safwat and Michael Kuehl \\ Department of Cardiology, UK
}

*Corresponding author: Viren Ahluwalia, Department of Cardiology, University

Hospitals Coventry and Warwickshire, UK.

\author{
Received Date: March 11, 2019 \\ Published Date: March 27, 2019
}

\section{Introduction}

Marantic endocarditis (MAE), "verrucous" or "Libman Sachs Endocarditis" are all different synonyms of the nowadays agreed term non-bacterial thrombotic endocarditis (NBTE), which is defined as fibrinous sterile valvular vegetations in the absence of infective endocarditis. MAE is associated with an autoimmunemediated inflammatory and thrombotic pathogenesis, but not with an infective pathogen. It is seen in conditions such as antiphospholipid syndrome and systemic lupus erythematosus (SLE), but also in terminal malignancies. The incidence of MAE is largely unknown. Small scale post mortem examinations reported NBTE to range between 0.9 to $1.6 \%$ [1]. This report aims to review current literature around MAE including presentation, diagnosis and management.

\section{Aetiology and Pathophysiology}

MAE is most commonly associated with malignancy with numerous MAE case reports in theliterature ranging from pancreatic cancer to adenocarcinoma of the lungs. Overall, adenocarcinomas have been shown to be mostly associated with MAE and of those mucin-secreting and pancreatic adenocarcinoma have the highest association [2,3]. It also has a well-documented association with systemic lupus erythematosus (SLE) and is associated with greater overall mortality in such patients [4]. Other associations include autoimmune disorders, hyper coagulate states, septicemia, severe burns, or chronic disease like tuberculosis, uremia or AIDS. The trigger for MAE and how it relates to commonly associated conditions is not well understood. The potential underlying pathophysiology might include endothelial injury in the setting of hypercoagulability [5]. This endothelial damage would then lead to primary and secondary clotting cascade activation involving platelets, clotting factors and inflammatory mediators leading thrombi formation and 'vegetation' on affected valves. It has a greater propensity to affect left sided valves.

\section{Presentation and Diagnosis}

MAE most commonly presents initially as a consequence of its distal embolic sequalae; Common complications include cerebrovascular events, such as strokes or transient ischemic attacks [6]. Emboli can ultimately affect any arterial system including critical limb ischaemia, splenic and renal infarcts and acute mesenteric ischaemia [3]. Localisation of causation can be linked to symptoms related to underlying aetiology. In patients with malignancy there maybe systemic constitutional symptoms including weight loss, night sweats and loss of appetite. Patients with SLE may describe a history of rashes, arthralgia and renal impairment, whilst those with antiphospholipid syndrome may describe recurrent deep vein thromboses or miscarriages.

Valvular vegetations in MAE are usually small, broad based and irregular shaped. Left sided (mitral more than aortic) and bilateral vegetations are more likely to represent MAE, than infective endocarditis (see picture). MAE are rarely associated with destructive changes to the heart valve. There is no definitive investigation in diagnosing MAE, with the key principle differential diagnosis being infective endocarditis. All patients should have a full work up including routine bloods to assess for organ dysfunction and serial blood cultures. Specific antibody (antiphospholipid antibodies) and auto-immune screen should be performed to rule autoimmune conditions such as SLE. As with conventional infective endocarditis imaging is crucial in proving MAE, with transesophageal echocardiography having much greater sensitivity over transthoracic [7] in demonstrating vegetations.

\section{Management}

There is a lack of evidence around the definitive way to manage MAE with most strategies extracted from case reports and small studies. This is in stark contrast to infective endocarditis, which has far clearer defined management principles [8]. In MAE key aspects of the underlying treatment relate to process aetiology and include management of the underlying condition likely causing MAE [9]. In the case of SLE this would include a mixture of corticosteroids and immunosuppression with agents including Azathioprine, Ciclosporin, or mycophentolate. Likewise, in patients with a malignancy these patients should be managed by a multidisciplinary 
team involving Cardiology and the oncology team Due to the highly prothrombotic nature of the condition, patients should be anticoagulated to prevent thromembolism. Unfractioned or low molecular heparin can be used as anticoagulant of choice. Novel anticoagulants like direct thrombin or factor Xa inhibitors have not been evaluated, nor have antiplatelets. Despite anticoagulation with a Vitamin K antagonist, recurrence of thromboembolic disease is common. Therefore, some investigators suggest anticoagulation with warfarin should be avoided and heparin should be used instead [10]. Surgery is not routinely recommended to treat MAE. However in cases of significant valvular dysfunction or individuals with recurrent thromboembolism despite management of the condition or anticoagulation, surgery can be considered, although definitive guidelines around which cases are appropriate are lacking.

The overall prognosis of patients with MAE is general poor as it is associated with disseminated and advanced underlying malignancy.

\section{Conclusion}

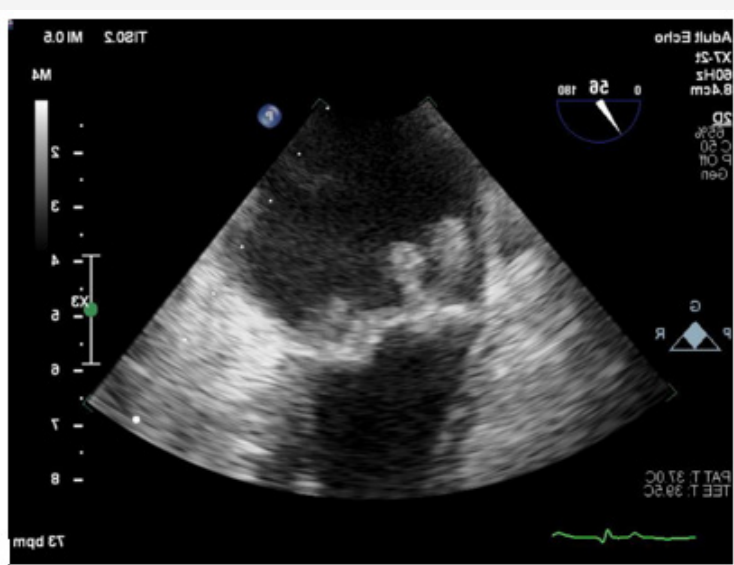

Figure 1: TOE Features: MAE most commonly affects the left side of the heart, commonly involving the basal and mid portion of the valve. Masses traditionally have irregular borders, variable echo density and an absence of independent motion.

Despite being well documented in numerous case reports there is still a very poor understanding around MAE compared to the more common infective endocarditis. Its aetiology remains highly theorized around a principle of hypercoagulability, with its link to conditions like SLE and malignancy being poorly understood. Management principles include imaging with echocardiography to confirm any 'typical' vegetations and negative serial blood cultures to rule out infective endocarditis followed by investigation and treatment of the underlying causative condition of marantic endocarditis. Palliative anticoagulation should be considered, although the overall outlook for patients with marantic endocarditis remains very poor. Future research into this condition might include register studies, comparison of novel anticoagulation with Vitamin $\mathrm{K}$ antagonists or studies into the effect of specific anti-tumor therapy on progression of marantic endocarditis (TOE Features) (Figure 1).

\section{Acknowledgement}

None.

\section{Conflict of Interest}

No conflict of interest.

\section{References}

1. Gonzalez Quintela A, Candela MJ, Vidal C, Roman J, Aramburo P, et al. (1991) Non-bacterial thrombotic endocarditis in cancer patients. Acta Cardiol 46(1): 1-9.

2. El Shami K, Griffiths E, Streiff M (2007) Nonbacterial thrombotic endocarditis in cancer patients: pathogenesis, diagnosis, and treatment. Oncologist 12(5): 518-523.

3. Borowski A, Ghodsizad A, Cohnen M, Gams E (2005) Recurrent embolism in the course of marantic endocarditis. Ann Thorac Surg 79(6): 21452147.

4. Moyssakis I, Tektonidou MG, Vasilliou VA, Samarkos M, Votteas V, et al (2007) Libman-Sacks endocarditis in systemic lupus erythematosus: prevalence, associations, and evolution. Am J Med 120(7): 636-642.

5. Deppisch LM, Fayemi AO (1976) Non-bacterial thrombotic endocarditis: clinicopathologic correlations. Am Heart J 92(6): 723-729.

6. Roldan CA, Sibbitt WL, Qualls CR, Jung RE, Greene ER, et al. (2013) Libman-Sacks endocarditis and embolic cerebrovascular disease. JACC Cardiovasc Imaging 6(9): 973-983

7. Roldan CA, Tolstrup K, Macias L, Qualls CR, Maynard D, et al. (2015) Libman-Sacks Endocarditis: Detection, Characterization, and Clinical Correlates by Three-Dimensional Transesophageal Echocardiography. J Am Soc Echocardiogr 28(7): 770-779.

8. Habib G, Lancellotti P, Antunes MJ, Bongiorni MG, Casalta JP, et al. (2015) 2015 ESC Guidelines for the management of infective endocarditis: The Task Force for the Management of Infective Endocarditis of the European Society of Cardiology (ESC). Endorsed by: European Association for Cardio-Thoracic Surgery (EACTS), the European Association of Nuclear Medicine (EANM). Eur Heart J 36(44): 3075-3128.

9. Ibrahim AM, Al Akchar M, Obaidi Z, Al Johany H (2018) Malignant Peritoneal Mesothelioma: A Rare Cause of Ascites. J Investig Med High Impact Case Rep 6: 2324709618807506.

10. Bell WR, Starksen NF, Tong S, Porterfield JK (1985) Trousseau's syndrome. Devastating coagulopathy in the absence of heparin. Am J Med 79(4): 423-430. 